\title{
Exchange interaction constants in CdCoSe diluted magnetic semiconductors
}

Cite as: Journal of Applied Physics 75, 4628 (1994); https://doi.org/10.1063/1.355911

Submitted: 29 September 1993 - Accepted: 08 December 1993 - Published Online: 17 August 1998

Jolanta Stankiewicz, Fernando Palacio and Javier Campo

\section{ARTICLES YOU MAY BE INTERESTED IN}

Diluted magnetic semiconductors

Journal of Applied Physics 64, R29 (1988); https://doi.org/10.1063/1.341700

Challenge us.

What are your needs for periodic signal detection?

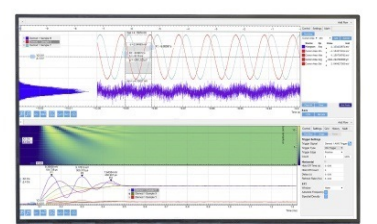

\section{0}

- Zurich

Instruments 


\title{
Exchange interaction constants in CdCoSe diluted magnetic semiconductors
}

\author{
Jolanta Stankiewicz \\ Centro de Física, Instituto Venezolano de Investigaciones Científicas, Apartado 21827, Caracas 1020A, \\ Venezuela \\ Fernando Palacio and Javier Campo \\ Instituto de Ciencia de Materiales de Aragon, CSIC-Universidad de Zaragoza, E-50009 Zaragoza, Spain
}

(Received 29 September 1993; accepted for publication 8 December 1993)

\begin{abstract}
The photoconductivity of diluted magnetic semiconductors $\mathrm{Cd}_{1-x} \mathrm{Co}_{x} \mathrm{Se}(0.011 \leqslant x \leqslant 0.041)$ has been measured at temperature of $1.6 \mathrm{~K}$ and in a magnetic field up to $6 \mathrm{~T}$. The photocurrent spectra show two peaks near the band-gap energy. They shift towards lower energies as the magnetic field intensity is increased. We interpret these lines in terms of free-exciton (exciton $A$ and $B$ ) dissociation. The large values of the observed shift arise from exchange interactions. A comparision of this shift with magnetization measurements done on the same samples allows us to estimate the exchange integrals for the CdCoSe system.
\end{abstract}

\section{INTRODUCTION}

Diluted magnetic semiconductors (DMS's) in which the magnetic atoms $(\mathrm{Mn}, \mathrm{Fe}, \mathrm{Co})$ randomly substitute the group II atoms of the host II-VI or III-V semiconductor (e.g., CdSe) are interesting for various reasons. ${ }^{1}$ They show novel physical effects related to the exchange interaction between the spins of localized electrons on the magnetic ions and the spins of band electrons. Their magnetic properties arising from the disordered nature of the magnetic sublattice are interesting as well. The DMS's with Mn as a substitutional atom have been most extensively studied up to now. The ground state of $\mathrm{Mn}^{2+}\left(3 d^{5}\right)$ ions in these alloys is magnetically active orbital singlet with no significant orbital momentum admixture. Therefore, the Mn-based DMS's behave as Brillouin-type paramagnets. Their magnetic and band properties are fairly well understood. ${ }^{1}$ More recently, there has been interest in Fe-based DMS materials. The $\mathrm{Fe}^{2+}\left(3 d^{6}\right)$ ions in II-VI compounds have only field-induced magnetic moments in the ground state. The Fe-based alloys exhibit Van Vleck-type paramagnetism and have properties very different from their $\mathrm{Mn}$-based counterparts. ${ }^{2-4}$ Only few studies have been reported on Co-based DMS system. The $\mathrm{Co}^{2+}$ $\left(3 d^{7}\right)$ has an orbital singlet ground state in a tetrahedrally coordinated lattice. An effective $g$-factor is larger than two $[g=2.295$ (Ref. 5)] due to a significant orbital momentum component in the ground state. The Co-based DMS's have a permanent magnetic moment and show Brillouin-type paramagnetism. Recently, results of electron Raman scattering, ${ }^{6}$ magnetic susceptibility, ${ }^{7,8}$ reflectance, ${ }^{9}$ magnetoreflectance, ${ }^{10-12}$ magnetization, ${ }^{11,12}$ and neutron scattering ${ }^{13}$ in Co-based DMS have been reported. They provide values of various exchange interaction parameters. However, no composition dependence of these parameters has been studied up to now.

In this paper we report photoconductivity and magnetization measurements in $\mathrm{Cd}_{1-x} \mathrm{Co}_{x} \mathrm{Se}(0.011 \leqslant x \leqslant 0.041)$ samples as a function of magnetic field $(0-6 \mathrm{~T})$. The results obtained allows us to estimate values of $s p-d$ exchange interaction constants in these alloys. Section II of the paper de- scribes the experimental procedure. The magnetic field dependence of the excitonic photocurrent and the determination of exchange integrals for CdCoSe system are discussed in Sec. III. The conclusions are stated in Sec. IV.

\section{EXPERIMENT}

$\mathrm{Cd}_{1-x} \mathrm{Co}_{x} \mathrm{Se}(0 \leqslant x \leqslant 0.05)$ single crystals used were grown by a modified Bridgman method. The composition, $x$, was checked by atomic absorption analysis. The values of $x$ were close to the nominal composition in all cases. X-ray diffraction analysis shows that $\mathrm{Cd}_{1-x} \mathrm{Co}_{x}$ Se crystals have a hexagonal structure. As-grown $n$-type samples with $x=0.011,0.024$, and 0.041 were studied. For photocurrent measurements samples were cleaved from the bulk material. Ohmic contacts were made with indium using ultrasonic soldering. The spectral response of photoconductivity was measured at $1.6 \mathrm{~K}$ in a magnetic field of up to $6 \mathrm{~T}$. Linearly or circularly polarized light in the Voigt configuration was used; the magnetic field was perpendicular or parallel to the crystal hexagonal $c$ axis. The magnetization was measured by an extraction method in magnetic fields up to 5 $\mathrm{T}$ at temperatures of 4.2 and $2.2 \mathrm{~K}$.

\section{RESULTS AND DISCUSSION}

Figure 1 shows how the normalized photocurrent in $\mathrm{Cd}_{0.99} \mathrm{Co}_{0.01} \mathrm{Se}$ varies with energy in the range corresponding to the energy gap. Each curve corresponds to a different value of the applied magnetic field. At $H=0$ two peaks in the photocurrent are observed. The lower-energy structure shows up only for $\mathbf{E} \perp c$ while the higher one is observed for both polarizations: $\mathbf{E} \perp c$ and $\mathbf{E} \| c(\mathbf{E}$ is the electric field vector of the incident radiation). Consequently, we interpret these structures in terms of dissociation of A-excitons (due to the top valence band $\Gamma_{9}$ in a wurtzite structure) and $B$-excitons (due to the crystal-field-split $\Gamma_{7}$ valence band). As the magnetic field intensity is increased, the peaks in the photocurrent spectra shift towards lower energy. The observed red shift of the exciton peaks arises from the exchange interaction and is therefore directly related to the 


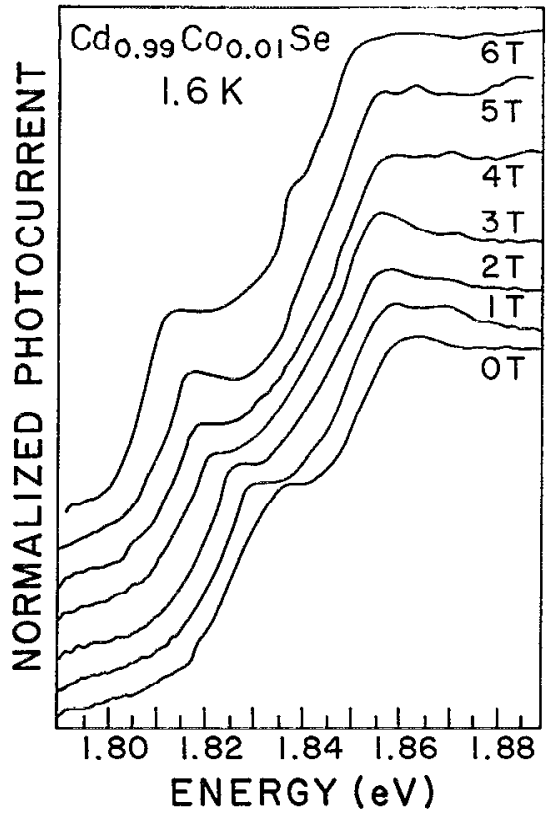

FIG. 1. Normalized photocurrent spectra in $\mathrm{Cd}_{0.99} \mathrm{Co}_{0.01} \mathrm{Se}$ at $T=1.6 \mathrm{~K}$ for various values of the magnetic field parallel to the $c$ axis.

magnetization of the material studied. The energy of the A-exciton can be well determined in the spectra; the structurc corresponding to the $B$-exciton is weaker and, therefore, there is a larger error in the determination of its energy.

Magnetization measurements results are shown in Fig. 2 for $T=2.2 \mathrm{~K}$ and for two different compositions of the CdCoSe system. The experimental data for $T=4.2$ and $2.2 \mathrm{~K}$ were fitted to the modified Brillouin function ${ }^{14}$

$$
M=M_{s} B_{3 / 2}\left(\frac{3 g \mu H}{2 k\left(T+T_{0}\right)}\right),
$$

where $M_{s}$ and $T_{0}$ are fitting parameters. $M_{s}$ reflects saturation value of the average spin component $S_{0}$ along magnetic field: $M_{s}=g \mu N_{0} x S_{0} ; \mu$ is the Bohr magneton, $N_{0}$ is the number of cation sites per gram, $k$ is Boltzmann's constant, and $g(=2.295)$ is the g-factor of the $\mathrm{Co}^{2+}$ ion. $B_{3 / 2}$ is Brillouin's function for spin $3 / 2, H$ is the magnetic field, and $x$ is the molar cobalt content. The solid lines in Fig. 2 are calculated using Eq. (1). We found that $S_{0}$ decreases from 1.26 to 0.963 and $T_{0}$ increases from 2.9 to $3.5 \mathrm{~K}$ as $x$ increases from 0.011 to 0.041 , respectively.

To relate photocurrent and magnetization data, we use results of band structure calculations which include the exchange interaction between band electrons and magnetic ions. ${ }^{15,16}$ Analytical solutions can be obtained for exciton splittings in wurtzite crystals in magnetic fields parallel and perpendicular to the $c$ axis. The shift of the A-exciton line in the $\mathbf{H} \| c$ geometry is

$$
\Delta E_{A}=\left(\alpha N_{0}-\beta N_{0}\right) x\left\langle S_{z}\right\rangle / 2,
$$

where $\alpha N_{0}$ and $\beta N_{0}$ are the exchange integrals for the conduction and the valence band, respectively, and $\left\langle S_{z}\right\rangle$ is the $\mathrm{Co}^{2+}$ mean spin in the field direction. The latter can be evaluated from magnetization measurements. The observed

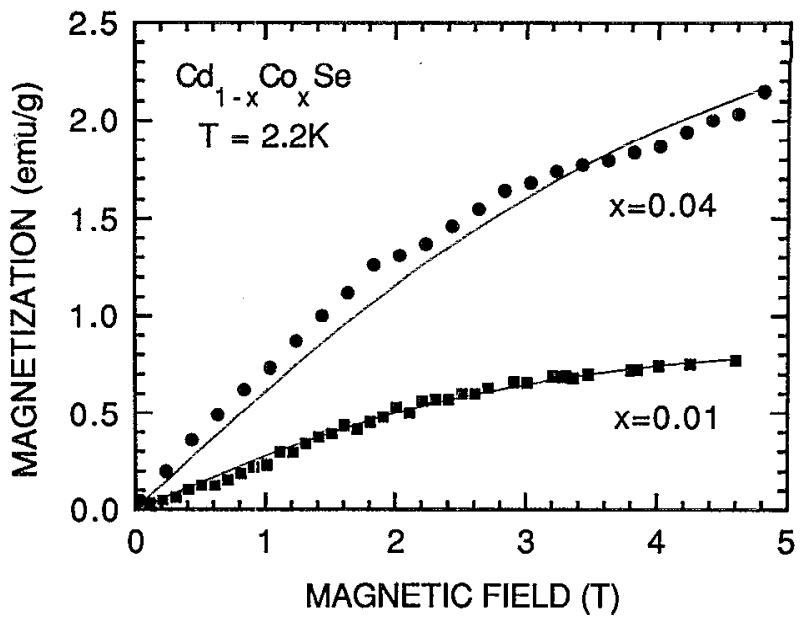

FIG. 2. Magnetization of two $\mathrm{Cd}_{1-x} \mathrm{Co}_{x}$ Se samples vs magnetic field at $T=2.2 \mathrm{~K}$ in a $\mathbf{H} \| c$ configuration.

$A$-exciton shift includes a contribution from the direct effect of applied magnetic field on the electron and hole comprising the exciton. In the field region studied, this contribution is small as compared to that of the $s p-d$ interaction and can be neglected. ${ }^{12}$

A plot of the A-exciton shift in a $\mathbf{H} \| c$ configuration versus $x\left\langle S_{z}\right\rangle$ is shown in Fig. 3 for the samples studied. Indeed, we find that the A-exciton splitting is proportional to the mean spin value as predicted by Eq. (2). A least-square fit yields a value for $\left(\alpha N_{0}-\beta N_{0}\right)$ of $(2.60 \pm 0.02) \mathrm{eV}$ for $x=0.011,(2.05 \pm 0.10) \mathrm{eV}$ for $x=0.024$, and $(1.94 \pm$ $0.06) \mathrm{eV}$ for $x=0.041$. The exchange constant $\beta N_{0}$ can be estimated independently from the variation of the energy separation between A- and B-exciton bands in the magnetic field. Assuming that the exchange constants for valence bands are isotropic and that band parameters of CdCoSe alloys are close to those of $\mathrm{CdSe}$, we obtain for $\alpha N_{0}$ a value of $(0.28 \pm 0.04) \mathrm{eV}$ for all values of the composition studied

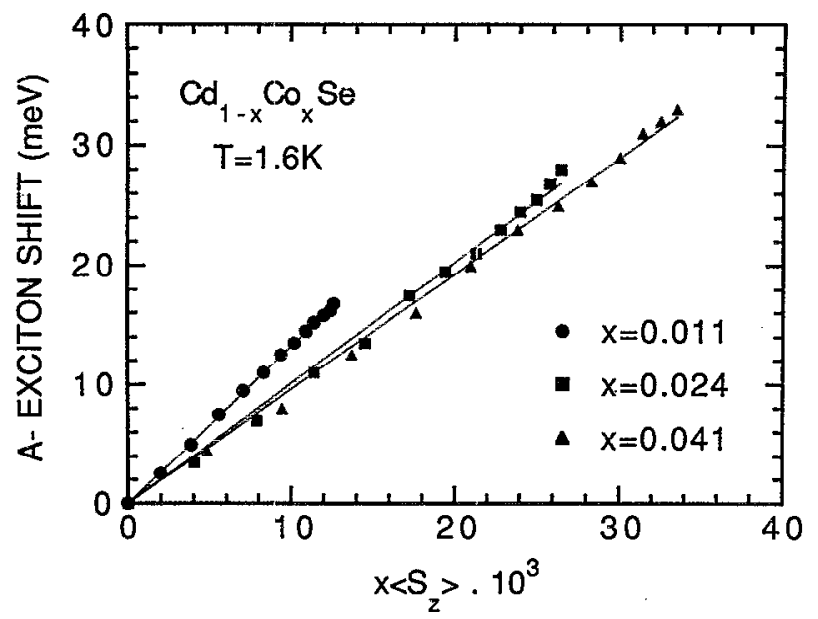

FIG. 3. A-exciton shift vs $x\left\langle S_{z}\right\rangle$ in a $\mathbf{H} \| c$ configuration for three different compositions of the $\mathrm{Cd}_{1-x} \mathrm{Co}_{x} \mathrm{Se}$ system. 


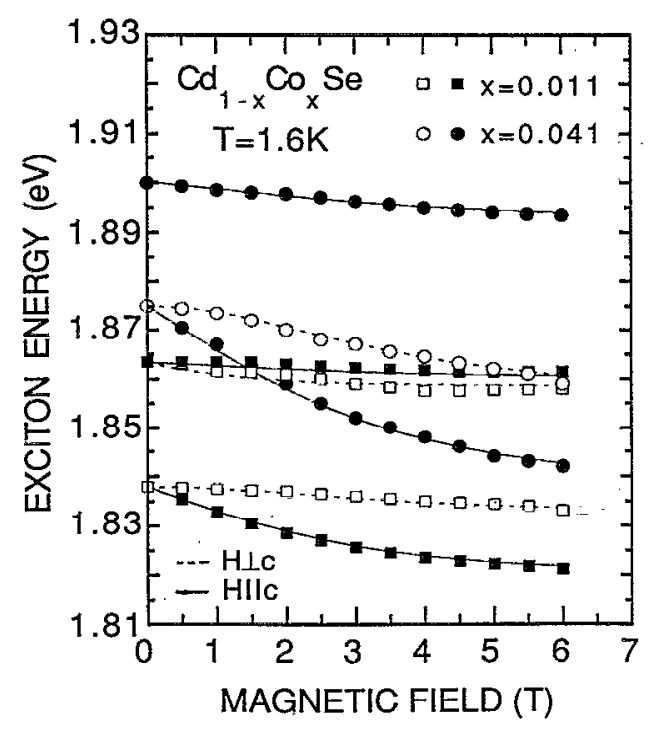

FIG. 4. Experimental (solid and open points) and calculated (solid and dashed lines) energy positions of $\mathrm{A}$ - and $\mathrm{B}$-exciton vs magnetic field intensity for $x=0.011$ and $x=0.041$ samples.

(we used the CdSe values for spin-orbit interaction constants ${ }^{17}$ and a value consistent with the zero-field data in our samples for noncubic crystal-field term).

Given band parameter and magnetization values, we can calculate optical transition energies and compare them with our photocurrent measurements as a function of magnetic field. Such comparison is shown, for $x=0.011$ and $x=0.041$, in Fig. 4. Solid (open) points represent excitonic transitions for $\mathbf{H} \| c(\mathbf{H} \perp c)$ configurations. The solid and dashed lines are calculated transitions energies using the values obtained for $\beta N_{0}$ and $\alpha N_{0}$. The agreement between experiment and calculated values is good.

The values that we have obtained for the exchange constants of CdCoSe system are very close to those reported previously for the same system ${ }^{11,12}$ and for $\mathrm{ZnCoSe}$ alloys. ${ }^{10}$ However, the values of $\left(\alpha N_{0}-\beta N_{0}\right)$, which are determined experimentally much more precisely, are almost twice as large as corresponding values of Mn-based DMS. This is primarily due to an increase in the $p-d\left(\beta N_{0}\right)$ exchange integral, since the values of $\alpha N_{0}$ vary by no more than $15 \%$ in different DMS. It has been shown that the $s p-d$ as well as $d-d$ exchange mechanisms in Mn-based DMS are related to hybridization between band electrons and $d$ electrons of the magnetic ion. ${ }^{18}$ Differences between $\alpha$ and $\beta$ constants reflect the different symmetry character of the conduction- and valence-band edges. Hybridization between $s$ (conductionband edge) and $d$ states is forbidden by symmetry, while the same mechanism introduces appreciable $d$ admixture to the anion-derived $p$ upper valence band. It leads to large negative values of $\beta$ and much smaller and positive values of $\alpha$. The superexchange interaction between magnetic ions $\left(J_{1}\right)$ also depends on $p$ - $d$ hybridization. Therefore, it seems worthwhile to compare values of different exchange constants in DMS. Table I shows available data for $\left(\alpha N_{0}-\beta N_{0}\right)$ and $J_{1}$ in DMS selenides. We choose for com-
TABLE I. Values of $\left(\alpha N_{0}-\beta N_{0}\right)$ and $J_{1}$ in DMS selenides.

\begin{tabular}{llclc}
\hline \hline System & $\left(\alpha N_{0}-\beta N_{0}\right)(\mathrm{eV})$ & Ref. & $J_{1}(\mathrm{~K})$ & Ref. \\
\hline CdMnSe & $1.37 \pm 0.07$ & 16 & $-10.6 \pm 0.2$ & 21 \\
CdFeSe & $1.90 \pm 0.3$ & 19,20 & -19.0 & 3 \\
CdCoSe & $2.5 \pm 0.1$ & 12 & $-37 \pm 5$ & 7 \\
ZnMnSe & $1.61 \pm 0.07$ & 4 & $-13.5 \pm 0.95$ & 23 \\
ZnFeSe & 1.96 & 24 & -22 & 25 \\
ZnCoSe & $2.42 \pm 0.04$ & 10 & $-54 \pm 8$ & 22 \\
\hline \hline
\end{tabular}

parison the same anion family since changes in the cation species are much less important in determining the magnetic propertics than changes in the anion species. ${ }^{18}$ Inspection of Table I shows clearly that there exists strong correlation between $\left(\alpha N_{0}-\beta N_{0}\right)$ and $J_{1}$ values in agreement with the predictions of the $s p-d$ hybridization model. It should be noted that $\beta N_{0}$ and $J_{1}$ exchange constants also depend on the relative position of the $d$ levels and the top of the valence band. This parameter varies significantly as one changes magnetic ions in DMS (in Mn-based DMS's it has a value of about $+3.5 \mathrm{eV}$ while in Fe-based DMS's it has a value of $-0.6 \mathrm{eV})$. Nevertheless, it seems to us that the $p$ - $d$ hybridization is much more important in exchange interactions.

If the hybridization is assumed to be independent of $x$, the calculated values of $\beta N_{0}$ and $J_{1}$ are nearly constant throughout the attainable composition range in DMS. Small variations in the magnetic ion-anion bond length may affect the hybridization amplitude and values of the exchange constants. Thus, a $13 \%$ increase in $\beta N_{0}$ and a $27 \%$ increase in $J_{1}$ has been predicted for $\mathrm{Cd}_{1-\mathrm{x}} \mathrm{Mn}_{\mathrm{x}}$ Te between $x=0$ and $x=0.75 .^{18}$ There is not sufficient data reported for $J_{1}$, but the available data for $\beta N_{0}$ in DMS selenides show an $x$ dependence diferent from the predicted one. In the $\mathrm{CdMnSe}$ system, $\beta N_{0}$ decreases by about $20 \%$ as $x$ increases from 0.05 to $0.38 .^{26,16,27}$ It decreases by about $40 \%$ in the $\mathrm{Cd}_{1-x} \mathrm{Fe}_{x} \mathrm{Se}$ alloys as $x$ varies from 0.011 to 0.07 (Refs. 19 and 20) and by about $20 \%$ in the $\mathrm{Cd}_{1-x} \mathrm{Co}_{x}$ Se system as $x$ goes from 0.011 to $0.04 .^{11,12}$ Similar behavior is observed in $\mathrm{ZnMnSe}$ alloys. ${ }^{4}$ In many cases, experimental accuracy is too poor to conclude how the exchange constants vary with $x$. However, the results presented in this paper as well as results published previously show that the valence band exchange constants depend on composition in many DMS. There is presently no explanation for such dependence.

Finally, we note that the experimental data for magnetization in $x=0.041$ samples deviate from the modified Brillouin function of spin $3 / 2$. The values obtained for the fitting parameter $S_{0}$ are smaller than the average spin component expected from the total number of $\mathrm{Co}^{2+}$ in the samples. The formation of antiferromagnetically ordered $\mathrm{Co}^{2+}$ clusters with no net magnetic moment can be responsible for this effect. Similar behavior has been reported for the CdMnSe and $\mathrm{CdFeSe}$ systems. The reduction in the magnetic moment of CdCoSe samples is larger than in Mn- or Fe-based alloys. This suggests that clustering effect is more important in the system studied. The other fitting parameter, $T_{0}$, is also much larger than that obtained for CdMnSe. This can be attributed 
to stronger antiferromagnetic interactions between more distant $\mathrm{Co}^{2+}$ ions.

\section{CONCLUSIONS}

The photoconductivity of $\mathrm{Cd}_{1-x} \mathrm{Co}_{x} \mathrm{Se}$ single crystals has been studied in magnetic fields at $1.6 \mathrm{~K}$. Near the bandgap energy, there are two peaks in the photocurrent whose energy positions are composition and magnetic-field dependent. We interpret these lines in terms of free exciton (exciton $A$ and B) dissociation. A comparison of the red shift of the photocurrent peaks with magnetization measurements done on the same samples allows us to determine the exchange integrals of the CdCoSe system. The values obtained are close to those obtained from magnetoreflection studies. They are much larger than corresponding values of the exchange constants in the Mn- or Fe-based DMS's. We attribute this to the increase in $p$ - $d$ hybridization in Co-based alloys. The previously reported large value of nearest neighbor exchange constant as well as relatively high values of effective temperatures yielded by fit of magnetization to Brillouin function support this conclusion. The exchange constant values for the valence band decrease with increasing Co content in our samples. Similar behavior has been observed in other DMS's. There is presently no explanation for such dependence.

\section{ACKNOWLEDGMENTS}

The authors would like to thank Dr. W. Giriat for providing the samples used. This work was partially supported by the Consejo Nacional de Investigaciones Cientificas $y$ Tecnológicas (of Venezuela). The work at the 1.C.M.A. was supported by CICYT Grant No. MAT91-681. One of us (J.C.) acknowledges a doctoral fellowship to the Departamento de Educacion y Cultura from Gobierno de Navarra.

\footnotetext{
${ }^{1}$ For a review, see Diluted Magnetic Semiconductors, edited by J. K. Furdyna and J. Kossut, Vol. 25 of Semiconductors and Semimetals (Academic, New York, 1988).
}

${ }^{2}$ D. Heiman, A. Petrou, E. D. Isaacs, S. H. Bloom, Y. Shapira, and W. Giriat, Phys. Rev. Lett. 60, 1876 (1988).

${ }^{3}$ A. Twardowski, J. Appl. Phys. 67, 5108 (1990).

${ }^{4}$ A. Twardowski, P. Glod, W. J. M. de Jonge, and M. Demianiuk, Solid State Commun. 64, 63 (1987).

${ }^{5}$ T. Hoshima, J. Phys. Soc. Jpn. 21, 1608 (1966).

${ }^{6}$ D. U. Bartholomew, E-K. Suh, A. K. Ramdas, S. Rodriguez, U. Debska, and J. K. Furdyna, Phys. Rev. B 39, 5865 (1989).

${ }^{7}$ A. Lewicki, A. I. Schindler, I. Miotkowski, and J. K. Furdyna, Phys. Rev. B 41, 4653 (1990).

${ }^{8}$ A. Lewicki, A. I. Schindler, J. K. Furdyna, and W. Giriat, Phys. Rev. B 40, 2379 (1989).

${ }^{9}$ J. Stankiewicz, J. Phys.: Condens. Matter 4, L145 (1992).

${ }^{10}$ X. Liu, A. Petrou, B. T. Jonker, J. J. Krebs, G. A. Prinz, and J. Warnock, J. Appl. Phys. 67, 4796 (1990).

${ }^{11}$ M. Nawrocki, F. Hamdani, J. P. Lascaray, Z. Golacki, and J. Deportes, Solid State Commun. 77, 111 (1991).

${ }^{12}$ M. Inone, N. Adachi, I. Mogi, G. Kido, and Y. Nakagawa, Physica B 184, 441 (1993).

${ }^{13}$ T. M. Giebultowicz, J. J. Rhyne, J. K. Furdyna, and P. Klosowski, J. Appl. Phys. 67, 5096 (1990).

${ }^{14}$ J. A. Gaj, R. Planel, and G. Fishman, Solid State Commun. 29, 435 (1979).

${ }^{15}$ S. I. Gubarev, Phys. Status Solidi B 134, 211 (1986).

${ }^{16}$ R. I. Aggarwal, S. N. Jasperson, J. Stankiewicz, Y. Shapira, S. Foner, B. Khazai, and A. Wold, Phys. Rev. B 28, 6907 (1983).

${ }^{17}$ C. L. Bir and G. E. Pikus, in Symmetry and Deformation Effects in Semiconductors (Nauka, Moscow, 1972).

${ }^{18}$ B. E. Larson, K. C. Haas, H. Ehrenreich, and A. E. Carlsson, Phys. Rev. B 37, 4137 (1988).

${ }^{19}$ A. Twardowski, K. Pakula, M. Arciszewska, and A. Mycielski, Solid State Commun. 73, 601 (1990).

${ }^{20}$ J. Stankiewicz and M. DiLorenzo, J. Appl. Phys. 69, 1479 (1991).

${ }^{21}$ J. Spalek, A. Lewicki, Z. Tarnawski, J. K. Furdyna, R. R. Galazka, and Z. Obuszko, Phys. Rev. B 33, 3407 (1986).

${ }^{22}$ A. Lewicki, A. I. Schindler, J. K. Furdyna, and W. Giriat, Phys. Rev. B 40, 2379 (1989).

${ }^{23}$ J. K. Furdyna, N. Samarth, R. B. Frankel, and J. Spalek, Phys. Rev. B 37, 3707 (1988).

${ }^{24}$ A. Twardowski, M. von Ortenberg, M. Demianiuk, and R. Pauthenet, Solid State Commun. 51, 849 (1984).

${ }^{25}$ A. Twardowski, A. Lewicki, M. Arciszewska, W. J. M. de Jonge, H. J. M. Swatgen, and M. Demianiuk, Phys. Rev. B 38, 10749 (1988).

${ }^{26}$ M. Arciszewska and M. Nawrocki, J. Phys. Chem. Solids 47, 309 (1986).

${ }^{27}$ I. Mogi, T. Kokubun, G. Kido, Y. Nakagawa, J. R. Anderson, W. Giriat, and M. Gorska, Physica B 177, 457 (1992). 